\title{
MODE- AND DIRECTION-DEPENDENT AKHIEZER DAMPING IN SINGLE- CRYSTAL SILICON RESONATORS
}

\author{
Srikanth S. Iyer ${ }^{1 *}$ and Rob N. Candler ${ }^{1,2}$ \\ ${ }^{1}$ Department of Electrical Engineering, University of California, Los Angeles, California, USA \\ ${ }^{2}$ California NanoSystems Institute, Los Angeles, California, USA
}

\begin{abstract}
In this work, we determine the intrinsic mechanical energy dissipation limit for single-crystal resonators due to anharmonic phonon-phonon scattering in the Akhiezer $(\Omega \tau \ll l)$ regime and evaluate the quality factor limit for intrinsic silicon devices. The energy loss is derived using perturbation theory and the linearized Boltzmann transport equation for phonons, and includes the direction- and polarization-dependent mode-Grüneisen parameters in order to capture the strain-induced anharmonicity among phonon branches. Our new theoretical framework reveals that Akhiezer damping, previously thought to depend only on material properties, has a strong dependence on resonant mode shape. Critically, we show that shear mode devices do, in fact, experience Akhiezer damping, which is in direct contrast to the result produced when applying existing models. Our expression satisfies the pressing need for a reliable analytical model that can predict the Akhiezer damping limits for modern resonant microelectromechanical systems, where precise manufacturing techniques and accurate finite-element methods can be used to select particular vibrational mode shapes and crystal orientations.
\end{abstract}

\section{INTRODUCTION}

The performance of mechanical resonators is governed by dissipation of energy stored in the resonant vibrational mode to other acoustic modes or the environment [1]. Despite the prevalence of resonant microelectromechanical systems (MEMS) as high-performance inertial sensors, mass-based chemical sensors, timing references and frequency filters, the dissipation in these structures is not well understood. The loss can be difficult to determine because there is no single, predictive theory to evaluate the quality factor $(Q)$, defined as $2 \pi[$ (energy stored)/(energy loss per cycle)]. Accurate prediction of $Q$ has tremendous design implications because it is directly related to device performance metrics including sensitivity for resonant sensors, bandwidth for radio-frequency filters, and phase noise for timing references.

This work focuses on determining intrinsic dissipation limits in dielectric and semiconductor crystals, which are governed by the interaction between the elastic wave and thermal phonons. This interaction has two components: spatial phonon transport and local phonon scattering. We focus on the local effect, Akhiezer damping, because it is present in all vibration modes and serves as an ultimate upper bound on the performance of MEMS resonators. The Akhiezer damping limit is particularly relevant for highfrequency and bulk-mode resonators, where the spatial effect (thermoelastic dissipation) is negligible [2]. Here, we derive an expression for Akhiezer loss that captures the effect of anharmonic phonon-phonon scattering as well as crystalline anisotropy.

In Akhiezer damping, strain produced by the mechanical wave modulates the phonon frequencies and, consequently, the local equilibrium phonon distribution. The phonon populations cannot change instantaneously and will relax towards the modulated equilibrium distribution via phonon-phonon scattering when the thermal relaxation time $(\tau)$ is significantly less than the period of the mechanical wave. Time varying strain and finite $\tau$ mean the time-dependent phonon populations lag behind their (perturbed) equilibrium value. This relaxation towards equilibrium is an entropy-producing process that consumes energy from the elastic wave. It is important to note that the Akhiezer damping model applies only when the scattering rate $(1 / \tau)$ is significantly larger than the frequency $(\Omega)$ of the mechanical vibration $(\Omega \tau \ll$ 1 ), which is the case at room temperature for commonly used acoustic materials such as silicon, germanium, and quartz [3].

This limit on mechanical energy dissipation was first described by Akhiezer [4] and later solved by Woodruff using the linearized Boltzmann transport equation (BTE) and the Debye approximation to arrive at a simplified, isotropic expression for internal friction $\left(Q^{-1}\right)$ involving only classical, bulk parameters [5].

$$
Q^{-1}=\frac{\gamma_{0}^{2} C_{v} T}{\rho c^{2}} \Omega \tau
$$

Here, $T$ is the ambient temperature, $\rho$ is the material density, $c$ is the Debye average sound velocity, $C_{v}$ is the specific heat per unit volume, and $\gamma_{0}$ is the average Grüneisen parameter associated with thermal expansion. Woodruff derives this result by assuming that all phonon modes are perturbed identically by the strain wave and neglecting the perturbation of the internal temperature of the solid. This expression is often used to make an order of magnitude prediction of the internal friction limit for a given material [6]. Modern devices, however, approach and even exceed Woodruff's oversimplified limit [7-10], indicating a need for a more predictive, analytical model that can be evaluated in a straightforward manner using known material constants and reliably compared with experimental results.

In this work, we rigorously derive an expression for the internal friction limit due to anharmonic phonon-phonon scattering. We solve for the energy loss using the analytically sound Boltzmann transport method, but rather than assuming all phonon modes are perturbed equally by strain, we include the directional- and polarization-dependent mode-Grüneisen parameters. The resulting expression for Akhiezer damping can still be evaluated using bulk parameters, but depends on the vibrational mode shape and crystal orientation of the resonator.

\section{ANHARMONIC ENERGY LOSS}

The derivation of the anharmonic phonon-phonon dissipation begins with the assumption that the strain wave is time harmonic with wave vector $\boldsymbol{K}$ and angular frequency $\Omega$ so that $\vec{\epsilon}(t) \propto$ $\exp [i(\boldsymbol{K} \cdot \boldsymbol{r}-\Omega \tau)]$, where $\boldsymbol{r}$ is the position vector. The strain perturbs the frequencies $(\omega)$ of all thermal phonons,

$$
\omega_{i}=\omega_{i 0}\left[1+\vec{\gamma}_{i} \cdot \vec{\epsilon}(t)\right]=\omega_{i 0}+\Delta \omega_{i}
$$

where the index $i$ denotes a pure-mode branch characterized by a direction and polarization along a crystal axis so that $\omega_{i}$ is the instantaneous phonon frequency of the $i^{\text {th }}$ branch and $\omega_{i 0}$ is the unperturbed equilibrium phonon frequency. Each high-symmetry direction in the Wigner-Seitz cell, the primitive Brillouin zone (BZ), has a longitudinal mode that is polarized along the direction of phonon propagation and two transverse modes that are polarized perpendicularly to the propagation direction. Fig. 1 shows the 13 principal crystallographic directions in the Wigner-Seitz cell for the diamond lattice. Strain, even in just a single direction, deforms the entire BZ, resulting in a perturbation of all phonon branches.

For cubic crystals, the strain tensor is assumed to be symmetric, so we can express it compactly as a vector $\vec{\epsilon}(t)$ with six 


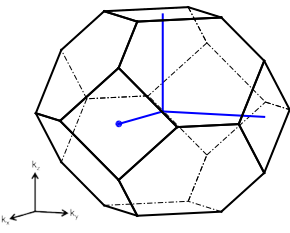

(a)

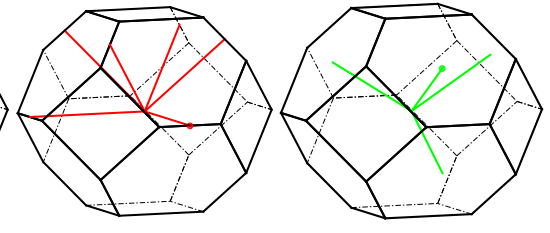

(b) (c)
Figure 1. Wigner-Seitz cell for the diamond lattice centered about the $\Gamma$ point. (a) Three $\langle 100\rangle$ directions (blue). (b) Six $\langle 110\rangle$ directions (red). (c) Four $\langle 111\rangle$ directions (green). There are 13 high-symmetry crystal directions and each contributes three pure modes, one longitudinal and two transverse, resulting in 39 distinct phonon branches.

components, denoted $\epsilon_{j}$, where the index $j$ indicates one of the six strain directions: three normal and three shear. Consequently, $\vec{\gamma}_{i}$ also has six components that are the mode-Grüneisen parameters $\gamma_{i, j}$, corresponding to the anharmonic perturbation of the $i^{\text {th }}$ branch frequency due to strain in the $j^{\text {th }}$ direction. Here, we assume that the $\gamma_{i, j}$ 's are independent of the phonon frequency and wavenumber. As a result of the phonon frequency perturbation, the instantaneous phonon population in each branch $N_{i}$ deviates from its thermal equilibrium Bose-Einstein distribution $N_{i 0}=\left(e^{\hbar \omega_{i 0} / k_{B} T}-1\right)^{-1}$, so that $N_{i}=N_{i 0}+\Delta n_{i}$.

We use the phonon BTE to describe the relaxation of the branch distributions, $N_{i}$. Akhiezer damping is a local effect, so we assume uniform strain and eliminate all spatial terms in the BTE. Thus, the relaxation towards equilibrium is solely determined by the scattering term. Following the approach of Woodruff and others, we employ the relaxation time approximation to describe the scattering term as the decay of $N_{i}$ towards a Bose-Einstein distribution $N_{i 0}^{\prime}$ at a modulated local temperature $T^{\prime}=\mathrm{T}+\Delta \mathrm{T}$ [11]. We can reduce the phonon BTE to

$$
\frac{\partial N_{i}}{\partial t}=\frac{N_{i}-N_{i 0}^{\prime}}{\tau}
$$

where we have characterized the relaxation process using the average phonon lifetime, $\tau$. Fig. 2 outlines the theoretical framework showing the perturbation of phonon frequencies and the corresponding dissipative relaxation.

The energy loss per cycle is simply the time average of the rate at which energy is lost via phonon-phonon scattering,

$$
U_{\text {loss per cycle }}=-\sum_{k, i}\left\langle H_{i} \cdot\left(\frac{\partial N_{i}}{\partial t}\right)_{\text {scatt }}\right\rangle_{\text {cycle }}
$$

where $H_{i}=\hbar \omega_{i}$ is the phonon Hamiltonian, $\langle\bullet\rangle_{\text {cycle }}$ denotes the time average over one period of the mechanical vibration, and the summation is over all possible wavenumbers $(k)$ and phonon branches $(i)$. Solving the BTE assuming plane wave solutions, $\Delta \omega_{i}, \Delta n_{i}, \Delta T \propto \exp [i(\boldsymbol{K} \cdot \boldsymbol{r}-\Omega \tau)]$, yields

$$
U_{\text {loss per cycle }}=\pi\left(\left\langle\gamma_{i, j}^{2}\right\rangle-\left\langle\gamma_{i, j}\right\rangle^{2}\right) C_{v} T \frac{\Omega \tau}{1+\Omega^{2} \tau^{2}}
$$

for uniaxial strain, where $\epsilon_{j}$ is the only nonzero strain component [12]. In the above expression, the angle brackets indicate an average of all phonon branches, and we have eliminated the cumbersome summation by assuming each branch contributes equally to the total phonon specific heat. Our energy loss expression depends not on the average Grüneisen parameter $\left(\gamma_{0}\right)$, but the difference between the mean-squared mode-Grüneisen parameter and the average mode-Grüneisen parameter squared, which reflects the fact that the phonon modes tend to relax towards the modified Bose-Einstein distribution determined by the perturbation of local temperature due to applied strain.

If the second- and third-order elastic coefficients are known, theoretical values for $\gamma_{i, j}$ can be obtained [3] and the energy-loss expression can be calculated using only bulk parameters. Ultimately, as we will show, the total energy loss can be expressed as a superposition of uniaxial losses.

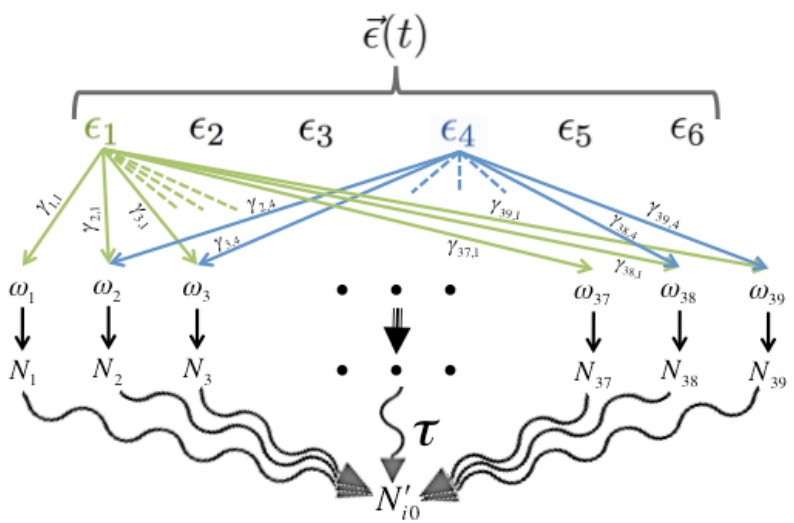

Figure 2. Overview of the energy loss due to anharmonic phononphonon scattering. Normal strains $\varepsilon_{1}-\varepsilon_{3}$ lead to a perturbation of phonon frequencies in each branch, characterized by the modeGrüneisen parameter $\gamma_{i, j}$. The modulation of phonon frequencies (and energies) means the respective branch populations $\left(N_{i}\right)$ are out of equilibrium. Each branch distribution relaxes towards the perturbed equilibrium distribution $\left(N_{i 0}^{\prime}\right)$ via phonon-phonon scattering, characterized by the phonon lifetime, $\tau$. The energy loss that occurs during this irreversible relaxation process is assumed to come from the acoustic wave, because that is the source of the perturbation. Shear strains $\varepsilon_{4^{-}} \varepsilon_{6}$, however, tend to result in reduced dissipation because only a fraction of the phonon branches are involved in the relaxation process, due to the restriction that volume is preserved under shear deformation.

\section{ENERGY STORAGE}

The quality factor is a ratio of energy stored to energy lost per cycle, so it is important to account for the anisotropy and mode dependence in both. For cubic crystals, the energy storage is anisotropic and depends on the deformation profile. The elasticity of a cubic crystal can be described by relating stress $(\vec{\sigma})$ and strain $(\vec{\epsilon})$ using the second-order elastic tensor.

For a given vibration mode and orientation, an effective Young's modulus or storage modulus $E_{\text {eff }}$ can be defined so that the energy storage per unit volume is $E_{\text {stored }}=\frac{1}{2} E_{\text {eff }} \epsilon_{0}^{2}$ [13]. The modes of interest are width extensional (WE), square extensional (SE), cubic extensional (CE), and Lamé; their deformation profiles and expressions for effective storage moduli are given in Table I. These are commonly used vibration modes for single-crystal silicon resonators oriented along [100] axes. The WE, SE, and CE modes are modes where the deformation of the solid is primarily due to extension (and contraction) along one, two, or three principal axes, respectively. The Lamé mode is a pure-shear mode with only one nonzero strain component.

Table I. Displacement profiles and elastic storage moduli for common vibration modes of a single-crystal rectangular parallelepiped with edges oriented along the [100] directions.

\begin{tabular}{c|c|c|c}
\hline $\begin{array}{c}\text { Width- } \\
\text { Extensional }\end{array}$ & $\begin{array}{c}\text { Square- } \\
\text { Extensional }\end{array}$ & $\begin{array}{c}\text { Cubic- } \\
\text { Extensional }\end{array}$ & $\begin{array}{c}\text { Lamé } \\
\text { (shear) }\end{array}$ \\
\hline & $\leftarrow$ & & \\
\hline$\frac{\left(c_{11}-c_{12}\right)\left(c_{11}+2 c_{12}\right)}{c_{11}+c_{12}}$ & $c_{11}+c_{12}-\frac{2 c_{12}^{2}}{c_{11}}$ & $\frac{c_{11}+2 c_{12}}{3}$ & $c_{44}$ \\
\hline \hline
\end{tabular}




\section{QUALITY FACTOR}

Using the definition of the quality factor, the energy storage expression in the previous section, and the energy loss in Eq. (5), we can write the quality factor as

$$
Q=\frac{E_{e f f}}{\left(\left\langle\gamma_{i, j}^{2}\right\rangle-\left\langle\gamma_{i, j}\right\rangle^{2}\right) C_{v} T} \frac{1+\Omega^{2} \tau^{2}}{\Omega \tau}
$$

for the uniaxial case. If we employ Woodruff's simplifications that the material is isotropic so all $\gamma_{i, j}=\gamma_{0}$ and that $\Delta T=0$, and assume the storage modulus is simply the bulk modulus $B=\rho c^{2}$, this expression reduces identically to Eq. (1) in the low-frequency limit. Woodruff's assumptions allow for simple estimation of $Q$ using bulk material data, but it is important to note that these assumptions are not self-consistent. If the material is assumed to be isotropic and $\gamma_{i, j}=\gamma_{0}$, then the average $\left\langle\gamma_{i, j}\right\rangle=\gamma_{0}$, which implies that $\Delta T \neq 0$. In fact, when this assumption is applied rigorously, $\left\langle\gamma_{i, j}^{2}\right\rangle=\left\langle\gamma_{i, j}\right\rangle^{2}=\gamma_{0}^{2}$ and the dissipation in Eq. (5) is zero. We note that the result derived here matches Zener's phenomenological form for quality factor due to anelastic relaxations in a solid [14], as expected, because the frequency dependence of the dissipation arises from a phase delay between the applied strain wave and the corresponding relaxation of the ensemble of thermal phonons.

\section{MODE-DEPENDENT ENERGY LOSS}

The expression in Eq. (6) accounts for the anisotropic and mode-dependent energy storage, but only includes loss due to uniaxial strain. In order to more accurately determine the losses, we define an effective mode-Grüneisen parameter $\gamma_{i \text {,eff }}$ as the weighted average of the components of $\vec{\gamma}_{i}$ by their corresponding strain component $\epsilon_{j}$. Thus, we can capture the perturbation of the phonon branch frequency due to strain in more than one direction.

Symmetry conditions in cubic crystals allow us to write $\left\langle\gamma_{i, e f f}\right\rangle=\alpha\left\langle\gamma_{i, 1}\right\rangle$ for pure extensional modes and $\left\langle\gamma_{i, e f f}\right\rangle=\alpha\left\langle\gamma_{i, 1}\right\rangle$ for pure shear modes, where $\alpha$ is a coefficient determined by the relative axial strain in the $\mathrm{x}, \mathrm{y}$, and $\mathrm{z}$ directions. The quality factor is reduced by a factor of $1 / \alpha^{2}$ [12]. In order to retain the simplicity of Eq. (1), we express the quality factor as

$$
Q=\frac{E_{e f f}}{\Gamma_{a}^{2} C_{v} T} \frac{1+\Omega^{2} \tau^{2}}{\Omega \tau}
$$

and define the anharmonic Grüneisen parameter as

$$
\Gamma_{a}^{2}=\alpha^{2}\left(\left\langle\gamma_{i, j}^{2}\right\rangle-\left\langle\gamma_{i, j}\right\rangle^{2}\right)
$$

Our expression shows that the quality factor depends distinctly on the resonant frequency, due to a mismatch between the period of the elastic wave and the phonon lifetime, and the strain profile, due to fundamental differences in the strength of the phonon frequency perturbation, which we quantify using $\alpha^{2}$.

Mason and Bateman establish that the mode-Grüneisen parameters can be determined from second- and third-order elastic moduli and calculate $\gamma_{i, 1}$ and $\gamma_{i, 5}$ for silicon and germanium [3]. Critically, they show that $\left\langle\gamma_{i, 1}\right\rangle \approx \gamma_{0}$ for extensional modes and $\left\langle\gamma_{i, 5}\right\rangle=0$ for shear modes, as expected. The final parameter, the phonon lifetime $(\tau)$, is determined using the definition of bulk thermal conductivity $\kappa \equiv \frac{1}{3} C_{v} c^{2} \tau$. This is, in effect, an average time constant over all phonon branches.

\section{RESULTS AND DISCUSSION}

We evaluate $Q$ for single-crystal, intrinsic silicon resonators and compare the Akhiezer-limited performance for common vibration modes. Fig. 3 shows the room temperature $f \times Q$ product as a function of the mechanical resonant frequency for the WE, SE, $\mathrm{CE}$, and Lamé modes of a [100]-oriented intrinsic silicon resonator evaluated using the expression in Eq. (7) along with Woodruff's result for reference and a number of experimental results from silicon resonators in the literature [7-10, 15-24]. Due to the quadratic dependence of $Q$ on resonant frequency, the curves remain constant up to $\sim 20.5 \mathrm{GHz}$, corresponding to the condition $\Omega \tau=1$. Again, we note that the Akhiezer damping model applies only when $\Omega \ll 1 / \tau$, so the results should only be interpreted below this value; at higher frequencies, the strain varies faster than the phonon scattering rate, so an alternate model, often called Landau-Rumer dissipation, should be used instead [25].

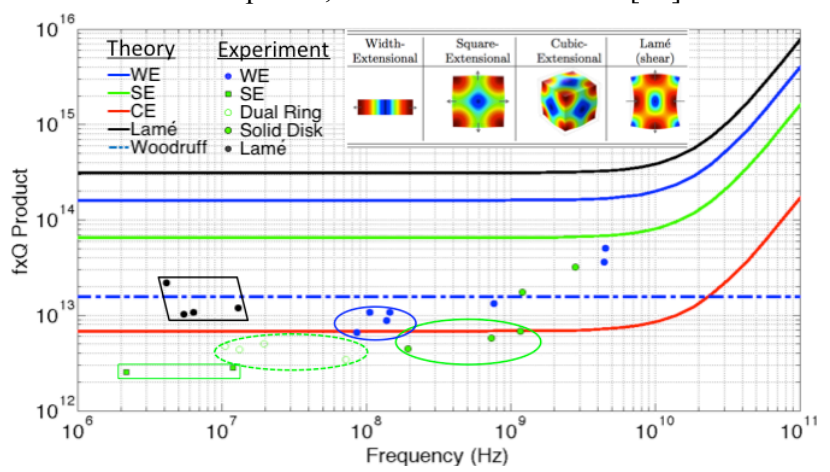

Figure 3. Anharmonic and anisotropic $f x Q$ product limits $v$ s. resonant frequency at room temperature for silicon. The theory curves (solid lines) indicate that Lamé mode resonators have the highest $Q$ limit, followed by the WE, SE and CE modes. The points are experimental results from high- $Q$ resonators in the literature [7-10, 15-24], a number of which exceed Woodruff's limit (dashed line), indicating that the simplified, isotropic expression does not provide sufficient accuracy. The ungrouped points are measurements of higher-order harmonics, so the assumption in this work of uniform strain is not directly applicable.

The (solid) theory curves show that the upper limit on $Q$ for the WE mode is greater than that of the SE mode, which is, in turn, larger than the $\mathrm{CE}$ mode. In the WE mode, Poisson contraction reduces the net phonon frequency perturbation compared to the uniaxial case. The SE and CE modes oppose the natural Poisson contraction leading to increased dissipation. Our results indicate that the quality factor limits for silicon at a specified resonant frequency can vary by more than an order of magnitude. The Lamé mode has the highest upper bound on $Q$ for the modes considered in this work, despite having the smallest energy storage modulus. For shear waves, phonon scattering is limited to volume-preserving phonon branches, leading to reduced energy dissipation because fewer branches, and less phonon energy, are subject to the relaxation process. We note that Woodruff's isotropic formula actually predicts infinite $Q$ (zero dissipation) for shear modes because the average Grüneisen parameter for volume-preserving modes is zero. The limitations of Woodruff's expression have been acknowledged in the past $[11,26]$, but we provide a viable alternative expression that shows that shear-mode vibrations do in fact lead to anharmonic phonon-phonon dissipation.

The evaluation of the anharmonic and anisotropic expression derived in this work indicates that Woodruff's order-of-magnitude result (dashed line) fails to provide an upper bound on the quality factor due to Akhiezer damping. In fact, several silicon resonators with quality factors that exceed Woodruff's limit have already been fabricated and measured in the literature [7-10], indicating the important need for our more accurate damping model that provides a robust upper bound on the performance of modern micromechanical resonators.

The experimental points are broadly categorized by geometry and mode type. The highest $Q$ resonators of a given type are grouped horizontally, reinforcing the assertion that the $f \times Q$ product is constant for a particular mode shape, in accordance with 
Eq. (7). The evaluation of Eq. (7) provides an upper bound on the quality factor, so it only predicts the performance of devices that are limited by anharmonic phonon-phonon dissipation, meaning other loss mechanisms including TED, air-damping, and anchor loss must have insignificant contributions. We also note that the theory lines in Fig. 3 use the idealized mode profiles in Table I, and, do not necessarily predict the exact behavior of the devices, because the actual vibrational modes are complicated functions of the geometry and boundary conditions of the structure. Additionally, the results here are for intrinsic silicon, and do not account for variations due to dopant species and density. The most accurate results can be obtained if the doping dependencies of second- and third-order elastic coefficients and thermal conductivity, which determines $\tau$, are known.

\section{CONCLUSION}

In this work, we provide an analytical expression for the quality factor due to anharmonic phonon-phonon dissipation that explicitly includes the anisotropic energy storage and loss in a cubic semiconductor or dielectric crystal. We provide a rigorous derivation of the anharmonic loss using the phonon BTE and introduce the important simplifications that must be made in order to facilitate quality factor calculation using known material parameters. These simplifications are presented and justified and evaluated for the most common vibration modes for [100] silicon. Our advanced model combined with relatively straightforward evaluation allows for meaningful comparisons between theory and experimental results and provides insight into the efficiency of different vibrational modes in the Akhiezer dissipation limit. Critically, we show explicitly that shear-mode vibrations do, in fact, experience Akhiezer damping, but have reduced dissipation in this limit because only a fraction of phonon branches are involved in the dissipative relaxation process. The formulations introduced in this work can be extended to account for doping dependence (when appropriate material data are available) and integrated into a finite-element solver to provide the most accurate predictions of phonon-phonon dissipation for arbitrary vibration profiles.

\section{ACKLOWLEDGEMENTS}

This work was supported by the Army Research Office under Contract No. W911NF-12-1-0190 and the Defense Advanced Research Projects Agency under Contract No. N66001-13-1-4032.

\section{REFERENCES}

[1] V. B. Braginsky et al., Systems with Small Dissipation, University of Chicago Press, Chicago, 1985.

[2] R. N. Candler, A. Duwel, et al., "Impact of geometry on thermoelastic dissipation in micromechanical resonant beams", J. Microelectromech. Syst. 15, 927 (2006).

[3] W. P. Mason, Physical Acoustics: Principles and Methods, edited by W. P. Mason, Academic Press Inc., New York, 1965, Vol. IIIB.

[4] A. Akhiezer, "On the absorption of sound in solids", J. Phys. USSR 1, 277 (1939).

[5] T. O. Woodruff and H. Ehrenreich, "Absorption of sound in insulators", Phys. Rev. 123, 1553 (1961).

[6] S. Ghaffari, S. A. Chandorkar, et al., "Quantum limit of quality factor in silicon micro and nano mechanical resonators", Nat. Sci. Rep. 3, 3244 (2013).

[7] G. Wu et al., "High Q single crystal silicon micromechanical resonators with hybrid etching process", IEEE Sens. J. 12, 2414 (2012).

[8] D. Weinstein and S. Bhave, "Internal dielectric transduction in bulk-mode resonators", J. Microelectromech. Syst. 18, 1401 (2009).
[9] S.-S. Li et al., "Micromechanical 'hollow-disk' ring resonators", in Proceedings of the 17th IEEE International Conference on Micro Electro Mechanical Systems (MEMS), 2004 (IEEE, New York, 2004), pp. 821-824.

[10] M. Ziaei-Moayyed and R. T. Howe, "Higher-order dielectrically transduced bulk-mode ring resonator with low motional resistance", in Proceedings of the IEEE International Frequency Control Symposium (FCS), Newport Beach, 2010 (IEEE, New York, 2010), pp. 19-24.

[11] H. H. Barrett and M. G. Holland, "Critique of current theories of Akhieser damping in solids", Phys. Rev. B 1, 2538 (1970).

[12] S. S. Iyer and R. N. Candler, "Mode- and direction-dependent mechanical energy dissipation in single-crystal resonators due to anharmonic phonon-phonon scattering," Phys. Rev. Applied 5, 034002.

[13] M. Hopcroft, W. D. Nix, and T.W. Kenny, "What is the Young's modulus of silicon?", J. Microelectromech. Syst. 19, 229 (2010)

[14] V. P. Mitrofanov, L. G. Ovodova, and V. S. Shiyan, "Longitudinal sound-wave attenuation in sapphire due to phonon-phonon interaction”, Fiz. Tverd. Tela (Leningrad) 22, 1545 (1980).

[15] L. Khine and M. Palaniapan, "High-Q bulk-mode SOI square resonators with straight-beam anchors", J. Micromech. Microeng. 19, 015017 (2009).

[16] J. E.-Y. Lee and A. A. Seshia, "5.4-MHz single-crystal silicon wine glass mode disk resonator with quality factor of 2 million", Sens. Actuators A 156, 28 (2009).

[17] S. Pourkamali, G. K. Ho, and F. Ayazi, "Low-impedance VHF and UHF capacitive silicon bulk acoustic-wave resonators - Part II: Measurement and characterization", IEEE Trans. Electron Devices 54, 2024 (2007).

[18] A. K. Samarao, G. Casinovi, and F. Ayazi, "Passive TCF compensation in high Q silicon micromechanical resonators", in Proceedings of the 23rd IEEE International Conference on Micro Electro Mechanical Systems (MEMS), 2010 (IEEE, New York, 2010), pp. 116-119.

[19] H. M. Lavasani et al., "A $145 \mathrm{MHz}$ low phase-noise capacitive silicon micromechanical oscillator", in Proceedings of the International Electron Devices Meeting, San Francisco, 2008 (IEEE, New York, 2008), pp. 1-4.

[20] J. E.-Y. Lee et al., "A singlecrystal-silicon bulk-acousticmode microresonator oscillator", IEEE Electron Device Lett. 29, 701 (2008).

[21] C. Tu and J. E.-Y. Lee, "Increased dissipation from distributed etch holes in a lateral breathing mode silicon micromechanical resonator", Appl. Phys. Lett. 101, 023504 (2012).

[22] H. Zhu, Y. Xu, and J. E.-Y. Lee, "Piezoresistive readout mechanically coupled Lamé mode SOI resonator with Q of a million", J. Microelectromech. Syst. 24, 771 (2015).

[23] J. Wang, Z. Ren, and C. T.-C. Nguyen, "1.156-GHz selfaligned vibrating micromechanical disk resonator", IEEE Trans. Ultrason. Ferroelectr. Freq. Control 51, 1607 (2004).

[24] J. R. Clark et al., "High-Q UHF micromechanical radialcontour mode disk resonators", J. Microelectromech. Syst. 14, 1298 (2005).

[25] L. Landau and G. Rumer, "Absorption of sound in solids", Phys. Z. Sowjetunion 11 (1937).

[26] A. Nowick and D. Berry, Anelastic Relaxation in Crystalline Solids, Academic Press, New York, 1972.

\section{CONTACT}

*S.S. Iyer, tel: +1-914-400-6573; ssiyer@ucla.edu 